\title{
Cooperative changes in solvent exposure identify cryptic pockets, conformational switches, and allosteric coupling.
}

\author{
Justin R. Porter $^{1}$, Katelyn E. Moeder ${ }^{1}$, Carrie A. Sibbald ${ }^{1}$, Maxwell I. Zimmerman ${ }^{1}$, \\ Kathryn M. Hart', Michael J. Greenberg ${ }^{1}$, Gregory R. Bowman ${ }^{1,3}$ \\ ${ }^{1}$ Department of Biochemistry \& Molecular Biophysics, Washington University School of \\ Medicine, 660 South Euclid Avenue, St. Louis, Missouri 63110, United States \\ ${ }^{2}$ Department of Chemistry, Williams College, 880 Main Street, Williamstown, MA 01267 \\ ${ }^{3}$ Department of Biomedical Engineering and Center for Biological Systems \\ Engineering, Washington University in St. Louis, One Brookings Drive, St. Louis, \\ Missouri 63130, United States \\ Email Contact: jrporter@wustl.edu
}

Proteins are dynamic molecules that undergo conformational changes to a broad spectrum of different excited states. Unfortunately, the low populations of these states make it difficult to determine their structures or functional implications. In recent years, computer simulations have begun to reach the timescales necessary to identify and characterize functionally-relevant excited states. However, this advance has uncovered a further challenge: without foreknowledge of which structural features are most important, it can be extremely difficult to identify the most salient features of large simulation datasets. We reasoned that functionally-relevant conformational changes are likely to result in large, cooperative changes to the surfaces that are available to interact with potential binding partners. To examine this hypothesis, we introduce a method that returns a prioritized list of potentially functional conformational changes by segmenting protein sequences into clusters of residues that undergo cooperative changes in their solvent exposure, along with the hierarchy of interactions between these groups. We term these groups exposons to distinguish them from other types of clusters that arise in this analysis and others. We demonstrate, using three different model systems, that this method identifies conformational switches, allosteric coupling, and known cryptic pockets-a feature of some excited conformations wherein a potentially-druggable concavity appears that is not present in existing structures. Our results suggest that key functional sites are hubs in the network of exposons. As a further test of the predictive power of this approach, we apply it to discover cryptic allosteric sites in two different enzymes. In vitro experiments confirm our predictions for both systems. Importantly, we provide the first evidence for a cryptic allosteric site in CTX-M-9 $\beta$-lactamase and demonstrate that this site exerts the most potent allosteric control over activity of any pockets found in $\beta$-lactamases to date. 